\title{
Addendum to:
}

\section{An energy model for the optimal design of linear continuum structures}

\author{
J.E. Taylor
}

\begin{abstract}
The original paper of the above title presents an analytical model for problems in the optimal design of linearly elastic continuum structures, where the material modulus tensor has the role of design variable. Both internal (strain) energy and the expression of generalized cost are represented conveniently there, in a form where the modulus tensor is transformed into vector coordinates. The general design of linear continuum structures is stated as a max-min problem. Optimality conditions for the transformed design problem have particularly simple form.

Both local properties, represented by the relative values of components of the modulus tensor, and the global distribution of structural resource (material) are variable in the design. With some modification to the original formulation, these separate aspects of design can be represented explicitly in the model. This modified form, which directly facilitates study of the role of local properties in the prediction of optimal design, and which ultimately serves as the basis for schemes to perform computational solution, is described and substantiated here.
\end{abstract}

Key words continuum structures, energy model, material design, variational formulation, minimum compliance

\section{1}

\section{Introduction}

While it may be appreciated in general terms that the form of problem statement for optimization problems is not unique, it is not usual to examine different mathematical formulations as a part of the approach to studies in design optimization. At the same time, generally one

Received February 7, 2000

\section{J.E. Taylor}

Department of Aerospace Engineering, University of Michigan, Ann Arbor, MI 48109, USA

e-mail: janos@umich.edu or another among valid formulations for a given problem may be more convenient, more revealing, or more broadly applicable than others. Thus one may choose according to convenience for the purpose at hand between the reciprocal formulations "minimum compliance design within an upper bound on the amount of available material" and "minimum amount of material design within an upper bound on compliance", as an example. Both of these equivalent (reciprocal) forms appear often in the structural optimization literature. To consider another aspect of formulation, the isoperimetric problem statement used in the "Energy Model" to represent the minimum potential energy form for equilibrium analysis offers some convenience compared to its conventional form, insofar as it provides directly for specification of the goal value of compliance. Quite distinct from these examples, for the issue of interest in this note the concern has to do with how well the formulation provides for the detailed description of the design variables, here the material modulus tensor. The ability to deal effectively with the prediction of material properties as a part of design optimization depends, more or less, on how those properties are represented in the problem formulation. Also, with respect to the task of producing computational solutions, the variational formulation is of interest both for the role it may play in providing mathematical support for computational approximation, and as well for the insight that may be gained about the structure of the scheme for computation through examination of different variational formulations.

The purpose of this brief note is to present a formulation for the continuum design problem elaborated from the one of the original "energy formulation" paper. The modification is obtained via the partitioning of the original design variable, i.e. the material modulus tensor, into two parts. Thus the design variables in the elaborated formulation are identified respectively with "local properties", which may be evaluated in terms of the relative values (at each point) of the components of the material modulus tensor, and the global distribution of material or structural resource. An additional field design variable is introduced in order to express the new formulation, and the relation between the now variationally separate measures of material property design and the 
global distribution of material is governed there by an additional constraint.

\section{2}

\section{The elaborated formulation}

The focus here is on that part of the formulation that applies to design optimization, i.e. the characterization of elastostatics given originally (Taylor 1998) remains unaffected. The design part of the problem statement, labeled [D2] in the original paper, is restated here as the starting point for this exposition,

$$
\max _{B_{\gamma}}\left\{\int_{\Omega} \sum_{\gamma} e_{\gamma} B_{\gamma} \mathrm{d} V\right\}
$$

subject to

$$
\int_{\Omega}\left(\sum_{\gamma} b_{\gamma} B_{\gamma}\right) \mathrm{d} V-R \leq 0, \quad 0<\underline{B}_{\gamma} \leq B_{\gamma} \leq \bar{B}_{\gamma},
$$

The argument of "max" measures total strain energy (for minimum compliance design), and $B_{\gamma}$ symbolizes the design variable. Coefficient $e_{\gamma}$ in [D2] is a function of the strain tensor (evaluated for equilibrium), and unit cost $b_{\gamma}$, local bounds $\underline{B}_{\gamma}, \bar{B}_{\gamma}$, and the measure of total available resource $R$ are data. The optimality condition for this problem was given as $\left(\bar{\kappa}_{\gamma}, \underline{\kappa}_{\gamma}\right.$, and $K$ symbolize, respectively, the multipliers on the local upper and lower bounds, and the global resource constraint),

$$
\left.-e_{\gamma}+\bar{\kappa}_{\gamma}-\underline{\kappa}_{\gamma}+b_{\gamma} K=0\right\} \quad x \in \Omega, \quad \gamma \in G_{\gamma} .
$$

Thus in regions of the structure where neither upper or lower bounds are active, the unit energies $e_{\gamma}$ are proportional to component unit costs $b_{\gamma}$.

For the expression of the elaborated formulation, the additional field variable $B(x)$ is introduced, along with a constraint that prescribes the relation between it and the measure $B_{\gamma}(x)$ of local constitution. The problem formulation that reflects independent variation of local properties and distribution of resource over the structure is represented now, in terms of their separate measures, as

$[\mathrm{M}] \max _{B_{\gamma} ; B}\left\{\int_{\Omega} \sum_{\gamma} e_{\gamma} B_{\gamma} \mathrm{d} V\right\}$,

subject to

$0<\underline{B}_{\gamma} \leq B_{\gamma} \leq \bar{B}_{\gamma}, \quad 0<\underline{B} \leq B \leq \bar{B}$,

$\sum_{\gamma} b_{L \gamma} B_{\gamma}-B \leq 0, \quad \int_{\Omega}(b B) \mathrm{d} V-R \leq 0$.
Here $b_{L \gamma}$ represent componentwise unit costs of material, and their relative values control the local structure of the optimal material, i.e. the user may force the optimal material to be orthotropic, isotropic, etc. according to the values prescribed for these unit cost coefficients. (Note that $b_{L \gamma}$ differs from the original $b_{\gamma}$ in that the latter has unit cost of local properties and global distribution represented all in the one set of parameters.) At the same time, pointwise unit cost $\mathrm{b}$ is identified with distribution over the structure of material resource. Presence in the model of this unit cost coefficient may be useful to predict optimal topology (Guedes and Taylor 1997), as an example of its application. Relative values of components $B_{\gamma}$ measure local constitution still, as was the case in [D2]. (Note that care must be taken with the prescription of values for the local bounds $\underline{B}_{\gamma}, \bar{B}_{\gamma}$, and $\bar{B}$, in order to avoid contradiction.)

Stationarity conditions with respect to field design variable $B$ and local constitution variables $B_{\gamma}$ are, respectively,

$-K_{L}+b K_{G}-\underline{\kappa}+\bar{\kappa}=0$,

$-e_{\gamma}+K_{L} b_{L \gamma}-\underline{\kappa}_{L \gamma}+\bar{\kappa}_{L \gamma}=0$,

where $K_{L}(x)$ and $K_{G}$ symbolize multipliers on the third and fourth constraints in [M]. Elimination of $K_{L}$ between (2) and (3) leads to

$e_{\gamma}=b_{L \gamma} b K_{G}-\left(\underline{\kappa}_{L \gamma}+b_{L \gamma \underline{\kappa}}\right)+\left(\bar{\kappa}_{L \gamma}+b_{L \gamma} \bar{\kappa}\right)$.

The relationship between the original and modified formulations is observed by comparison of (1) and (4); thus the problems represented in them are equivalent for the corresponding values,

$b_{\gamma} K=b_{L \gamma} b K_{G}, \quad \underline{\kappa}_{\gamma}=\underline{\kappa}_{L \gamma}+b_{L \gamma} \underline{\kappa}$,

$\bar{\kappa}_{\gamma}=\bar{\kappa}_{L \gamma}+b_{L \gamma} \bar{\kappa}$.

The first equation (5) may be interpreted as the requirement that in the case where local constraints are not active, the component unit energies $e_{\gamma}$ of the optimal design will have identical values. The relation can be reduced further by comparison of the global resource constraints between the two formulations. In order that total "cost" represented in these constraints for the two problems is the same, unit cost coefficients must be related according to

$b_{\gamma}=b_{L \gamma} b$.

It follows from (6) and the first of (5) that multipliers $K$ and $K_{G}$, measuring unit energy per unit cost, have equal value. Of course (6) in turn implies a further restriction on the multipliers for the local bound constraints in the second and third equations (5). Finally, the next to 
last constraint in $[\mathrm{M}]$ is active at the solution, and this dictates the relation: between the optimal design results $B$ and $B_{\gamma}$, namely,

$\sum_{\gamma} b_{L \gamma} B_{\gamma}-B=0$

With these observations, the relation between the modified formulation and the original one is fully established.

To motivate one additional step in the modelling, recall that the form of material is established from the relative values of the design components $B_{\gamma}$.

Thus the characterization of local properties can be expressed in terms of the normalized set, say $\hat{B}_{\gamma}$, normed e.g. according to

$\frac{1}{N} \sum_{1}^{N} \hat{B}_{\gamma}=1$

All materials of similar constitution are identified via a scale factor, say $r$, per

$B_{\gamma}=r \hat{B}_{\gamma}$.

This expression is substituted into the relation between design variables $B$ and $B_{\gamma}$ to find

$B=\sum_{\gamma} b_{L \gamma} B_{\gamma}=r \sum_{\gamma} b_{L \gamma} \hat{B}_{\gamma}$.

Thus the value determined for $r$ by

$r=B /\left(\sum_{\gamma} b_{L \gamma} \hat{B}_{\gamma}\right)$

provides for the scaling of a structure of given local constitution $\hat{B}_{\gamma}$ to a known distribution of resource $B$. Such scaling may be required as part of a stepwise procedure for the computational solution of problem $[\mathrm{M}]$, for example.

\section{3}

\section{Discussion}

The relation between local structural characteristics and overall design appears as an essential aspect of modelling in the two historically significant areas of Michell truss design (Michell 1904; Prager 1974; Prager and Rozvany 1977) and the design of grillages (Rozvany 1976). The same feature shows up in one or another specific design setting in the developments reported by Olhoff et al. (1997), Pedersen (1993), Rozvany et al. (1982), and Foldager (1999), as examples. The formulation of the present note may be viewed simply as a generalization of the ideas from these earlier studies, as it applies in general to design of the linearly elastic continuum structure having unrestricted local properties.
Some insight may be gained about the relationship between the present energy model and earlier treatments of continuum design where the modulus tensor appears as the design variable, by consideration of the following two points. First, in the presentation of Bendsøe et al. (1994) for optimal design of the unrestricted modulus tensor, the unit cost of material is presumed to be of specific form, namely proportional to the trace or the Frobenius norm of the modulus tensor, and to be uniform over the structure. Neither limitation applies in the energy model for continuum design, i.e. the unit cost is expressed in general form and both local structure and the distribution of resource are treated as variable over the region of the structure. Somewhat surprisingly, it proves to be possible still with the generalized model to predict optimal local structure analytically, as it was in the earlier special case (where the optimal material proved to be uniformly orthotropic). On a quite different aspect of the modelling, in the cited earlier paper an interpretation is given where the operation max with respect to design is expressed in the form of a $\max \max$ sequence of operations, applying respectively to local properties and to distribution of resource (this simply reflects the property that the global "max" relies on pointwise maximization of the argument of the integral objective). In contrast, the identification with local properties and global distribution in $[\mathrm{M}]$ is accomplished through a reinterpretation of the prior design variable into the set of two variationally separate design variables. An interpretation to maximizate separately with respect to the two aspects of design, as was indicated by Bendsøe et al. (1994), is still possible within $[\mathrm{M}]$. Such considerations arise often in connection with implementation of analytical modelling into means for carrying out computational solutions. These and other issues related to computational treatment of the generalized design problem are to be described elsewhere.

Acknowledgements The research effort leading to the results reported here was facilitated in part using much appreciated support received from the Scientific Research Laboratories, Ford Motor Company.

\section{References}

Bendsøe, M.P. 1995: Optimization of structural topology, shape, and material. Berlin, Heidelberg, New York: Springer

Bendsøe, M.P.; Guedes, J.M.; Haber, R.B.; Pedersen, P.; Taylor, J.E. 1994: An analytical model to predict optimal material properties in the context of optimal structural design. $J$. Appl. Mech. 61, 930-937

Foldager, J.P. 1999: Design of composite structures. Ph.D. Dissertation, Special Report No. 39, Institute of Mechanical Engineering, Aalborg University, Denmark

Guedes, J.M.; Taylor, J.E. 1997: On the prediction of material properties and topology for optimal continuum structures. Struct. Optim. 14, 193-199 
Michell, A.G.M. 1904: The limits of economy of material in framed structures. Phil. Mag. 6, 589-597

Olhoff, N.; Jacobsen, J.B.; Ronholt, E. 1997: Three-dimensional structural topology and layout optimization based on optimum microstructures. Extended Abstracts of WCSMO-2 (held May 26-30 in Zakopane, Poland)

Pedersen, P. 1993: Optimal orientation of anisotropic materials. Optimal distribution of anisotropic materials. Optimal shape design with anisotropic material. In: Rozvany, G.I.N. (ed) Optimization of large structural systems (Proc. NATO/DFG ASI held in Berchtesgaden, Germany, 1991), pp. 649-682. Dordrecht: Kluwer
Prager, W. 1974: Introduction to structural optimization (Course No. 212, International Center for Mechanical Sciences, Udine). Vienna: Springer

Prager, W.; Rozvany, G.I.N. 1977: Optimization of structural geometry. In: Bednarek, A.R.; Cesari, L (eds.) Dynamical systems, pp. 265-293. New York: Academic Press

Rozvany, G.I.N. 1976: Optimal design of flexural systems. Oxford: Pergamon Press

Rozvany, G.I.N.; Olhoff, N.; Cheng, G.; Taylor, J.E. 1982: On the solid plate paradox in structural optimization. J. Struct. Mech. 10, 1-32

Taylor, J.E. 1998: An energy model for the optimal design of linear continuum structures. Struct. Optim. 16, 116-127 